\title{
PURE DESCENT FOR THE MODULE OF ZARISKI DIFFERENTIALS
}

\begin{abstract}
ERICH PLATTE
ABSTRACT. It will be shown that for any given pure extension $A \rightarrow B$ of noetherian $\boldsymbol{k}$-algebras, with $\boldsymbol{k}$ being a field of characteristic zero, and for any prime ideal $\mathfrak{p} \subseteq A$ the Zariski-Lipman conjecture for $A_{\mathfrak{p}}$ is solvable, if $B$ is a locally factorial domain for which the finite differential module is reflexive. We will also discuss an embedding property with respect to the module of Zariski differentials of $A_{\mathfrak{p}}$.
\end{abstract}

1. Preliminaries. A homomorphism $\varphi: R \rightarrow S$ of rings is defined to be pure, if for any $R$-module $M$ the canonical map $M \rightarrow M \otimes_{R} S$ is injective. If, for instance, $R$ is a direct $R$-summand of $S$ (via $\varphi$ ), then $\varphi$ is pure. If the extension $R \rightarrow S$ is pure, all ideals in $R$ are contracted, and therefore noetherianness and normality descend. We say that $\varphi: R \rightarrow S$ is pure in a prime ideal $q \subseteq S$, if the induced homomorphism $R_{\mathfrak{p}} \rightarrow S_{\mathfrak{q}}, \mathfrak{p}:=\varphi^{-1}(q)$, is pure. If $R$ is a local ring, the purity of $\varphi: R \rightarrow S$ is equivalent to saying that $\mathbf{E} \rightarrow \mathbf{E} \otimes_{R} S$ is injective, where $\mathbf{E}$ denotes the injective hull of the $R$-module $R / \mathrm{m}_{R}$, see (6.11) in [3]. Let $x \in \mathbf{E}$ generate the one-dimensional socle of the $R$-module $\mathbf{E}$ and $\mathrm{c}:=\operatorname{Ann}_{S}(x \otimes 1)$ with $\mathfrak{m}_{R} S \subseteq \mathrm{c} \subseteq S$. Then $\varphi$ is pure if and only if $c \neq S$, and in this case there exist prime ideals $q \subseteq S$ precisely those which contain $c-$ such that $\varphi$ is pure in $q$, i.e. the induced homomorphism $R \rightarrow S_{\mathrm{q}}$ is pure, too.

Secondly, we will discuss a class of pure extensions in characteristic zero. Let $\varphi: R \rightarrow S$ be a local homomorphism of local noetherian rings, and let $\varphi$ be nondegenerate in the following sense:

(i) $S$ satisfies the chain condition for prime ideals, i.e., if $\mathfrak{Q}, \mathfrak{Q}^{\prime}$ are prime ideals in $S$ with $\mathfrak{Q} \subseteq \mathfrak{Q}^{\prime}$, then $\operatorname{codim} \mathfrak{Q}+\operatorname{codim} \mathfrak{Q}^{\prime} / \mathfrak{Q}=\operatorname{codim} \mathfrak{Q}^{\prime}$, and

(ii) the equality $\operatorname{dim} R+\operatorname{dim} S / \mathfrak{m}_{R} S=\operatorname{dim} S$ holds.

If furthermore $R$ and $S$ are analytically normal domains over a field $k$ of characteristic zero, then $\varphi$ is pure.

Proof. We may assume that $R=\operatorname{proj} \lim R / \mathrm{m}_{R}^{i}$ and $S=\operatorname{proj} \lim S / \mathrm{m}_{S}^{i}$ are complete and that $k$ is a field of representatives of $R$. There exists a field $K$ of representatives of $S$ containing $k$. The extension $R \rightarrow R \hat{\otimes}_{k} K$ is faithfully flat, and the extended homomorphism $R \hat{\otimes}_{k} K \rightarrow S$ is nondegenerate. Therefore we may even assume $k=S / \mathrm{m}_{s}$. Now, let $r:=\operatorname{dim} S / \mathrm{m}_{R} S$ and $t_{1}, \ldots, t_{r}$ be elements of $\mathfrak{m}_{S}$ whose residue classes modulo $\mathfrak{m}_{R} S$ form a system of parameters in $S / \mathfrak{m}_{R} S$. The extended homomorphism $R^{\prime}:=R \llbracket T_{1}, \ldots, T_{r} \rrbracket \stackrel{\varphi^{\prime}}{\rightarrow} S$ with $\varphi^{\prime} \mid R=\varphi$ and

Received by the editors January 30, 1980 and, in revised form, March 24, 1980.

1980 Mathematics Subject Classification. Primary 13B02; Secondary 14B15. 
$T_{i} \mapsto t_{i}$ is finite and injective by reasons of dimension. Since $\operatorname{rank}_{R^{\prime}} S$ is a unit in $k$, the canonical trace gives a projection $\pi: S \rightarrow R^{\prime}$ such that $\pi \circ \varphi^{\prime}$ is the identity on $R^{\prime}$. Therefore $R^{\prime}$ is a direct $R^{\prime}$-summand of $S$ (via $\varphi^{\prime}$ ), hence $\varphi^{\prime}$ is pure. Since $R \rightarrow R^{\prime}$ is faithfully flat, the assertion follows. In particular, the conditions (i) and (ii) imply:

(1) The height of a prime ideal in $R$ coincides with the height of its extension in $S$.

(2) Any prime ideal of height $h$ in $S$ contracts to one of height $<h$ in $R$.

(3) For any prime ideal $q \subseteq S$ the homomorphism $\varphi: R \rightarrow S$ is nondegenerate in $q$, i.e. the local homomorphism $R_{\mathfrak{p}} \rightarrow S_{\mathfrak{q}}, \mathfrak{p}:=q \cap R$, is nondegenerate.

In summary: If $\varphi: R \rightarrow S$ is a nondegenerate local homomorphism of excellent normal local rings over a field of characteristic zero, then $\varphi$ is pure and nondegenerate in any given prime ideal $\mathrm{q} \subseteq S$.

We outline the proof of (1); the assertions (2) and (3) are easy consequences. Let $\mathfrak{p}$ be a prime ideal of height $c$ in $R$. Since $R_{\mathfrak{p}} \rightarrow S_{\mathfrak{p}}$ is pure, $\mathfrak{p} S_{\mathfrak{p}}$ is not the unit ideal, and therefore

$$
\mathrm{c}=\operatorname{dim} R_{\mathfrak{p}}>\operatorname{codim} \mathfrak{p} S_{\mathfrak{p}}>\operatorname{codim} \mathfrak{p} S .
$$

Now assume c $>\operatorname{codim} \mathfrak{p} S$. Let $\mathfrak{q} \subseteq S$ be a minimal prime ideal of $\mathfrak{p} S$ with $\operatorname{codim} q=\operatorname{codim} \mathfrak{p} S$. There exist elements $g_{1}, \ldots, g_{r-c}, r:=\operatorname{dim} R$, such that $\mathfrak{p}+\sum_{i=1}^{r-c} R g_{i}$ is a $\mathfrak{m}_{R}$-primary ideal in $R$. It follows $\operatorname{codim}\left(q+\sum_{i=1}^{r-c} S g_{i}\right)>r$. If c $>\operatorname{codim} q$, this contradicts Krull's principal ideal theorem for the ring $S / \mathfrak{q}$.

All rings are considered to be commutative with 1 , all modules (and algebras) are unitary. If $\mathfrak{p}$ is a prime ideal in the ring $R$, we denote by $\kappa(\mathfrak{p})$ the residue field of $R_{\mathfrak{p}}$. If $R$ is a local ring, the maximal ideal of $R$ is denoted by $\mathfrak{m}_{R}$.

2. Pure extensions and differential descent. In this section let $k$ be a field of characteristic zero and $\varphi: A \rightarrow B$ be a local homomorphism of local noetherian $k$-algebras for which the finite differential modules $\mathrm{D}_{k}(A)$ and $\mathrm{D}_{k}(B)$ exist. For the theory of finite differential modules we refer the reader to [8]. Assume that $a \subseteq B$ is a prime ideal such that

(1) $B_{\mathrm{q}}$ is a normal domain and $\mathrm{D}_{k}(B)_{\mathrm{q}}$ is reflexive, and

(2) $\varphi$ is pure in $q$.

Let $\mathfrak{p}:=\varphi^{-1}(\mathfrak{q})$ and $V=\mathrm{D}(\mathfrak{a}) \subseteq X:=$ Spec $A_{\mathfrak{p}}$ be the regular locus of $A_{\mathfrak{p}}$ with an ideal $\mathfrak{a} \subseteq A_{\mathfrak{p}}$ of height $\geqslant 2$. Let $Y$ denote the affine scheme Spec $B_{\mathfrak{q}}$ and $\varphi_{*}: Y \rightarrow X$ the induced morphism of affine schemes. We denote by $U \subseteq Y$ the open set $\varphi_{*}^{-1}(V)$ and by $Z \subseteq Y$ the closed subset $Y \backslash U$.

If $Z \neq \varnothing$, then $a$ is not the unit ideal, and therefore $V$ is not affine. It follows that $U=\varphi_{*}^{-1}(V)$ is not affine, too, since for any $A_{\mathfrak{p}}$-module $M$ the canonical homomorphisms

$$
\mathbf{H}^{i}(V, \tilde{M}) \rightarrow \mathbf{H}^{i}\left(U, \widetilde{M \otimes_{A_{\mathrm{v}}} B_{\mathrm{q}}}\right), \quad i>0,
$$

are injective; see $\$ 6$ in [3].

The methods which we use in the proof of the following proposition are similar to those of (3.1) in [6]. 
Proposition 1. If $\operatorname{codim}_{Y} Z>2$, then there exist (at least) $s:=\operatorname{dim} A_{\mathfrak{p}}$ elements $f_{1}, \ldots, f_{s} \in \mathfrak{p}$, whose differentials $\mathrm{d} f_{1}, \ldots, \mathrm{d} f_{s}$ form part of a minimal system of generators of $\left(\mathrm{D}_{k}(A)\right)_{\mathfrak{p}}^{* *}$ via the canonical homomorphism $\mathrm{D}_{k}(A)_{\mathfrak{p}} \rightarrow\left(\mathrm{D}_{k}(A)\right)_{\mathfrak{p}}^{* *}$.

Proof. Let $f_{1}, \ldots, f_{t}$ be elements of $\mathfrak{p}$, which minimally generate a minimal differential reduction $\mathfrak{b}^{\prime} \subseteq B_{\mathrm{a}}$ of the ideal $\mathfrak{b}:=\mathfrak{p} B_{\mathfrak{q}}-$ for this term see [7]-i.e. the differentials $\mathrm{d} f_{1}, \ldots, \mathrm{d} f_{t}$ with $f_{i} \in \mathfrak{p}$ are a minimal system of generators of the $B_{\mathrm{q}} / \mathfrak{b}$-module

$$
\mathrm{U}(\mathfrak{b}):=B_{\mathfrak{q}} \mathrm{d}_{\mathfrak{a}} \mathfrak{b} / \mathfrak{b D} D_{k}(B)_{\mathfrak{a}}
$$

In (8.11) of [8] G. Scheja and U. Storch have shown that $\mathfrak{b}$ is integrally dependent on $\mathfrak{b}^{\prime}$, i.e. there exists an integer $n$ with

$$
\mathfrak{b}^{\prime} \cdot \mathfrak{b}^{n}=\mathfrak{b}^{n+1} \text {. }
$$

Since $\mathfrak{b}^{\prime}=\left(\dot{f}_{1}, \ldots, f_{t}\right) \cdot B_{\mathfrak{q}}$ and $\mathfrak{b}=\mathfrak{p} B_{\mathfrak{q}}$ are extended ideals, we obtain that

$$
\left(f_{1}, \ldots, f_{t}\right) A_{\mathfrak{p}} \cdot\left(\mathfrak{p} A_{\mathfrak{p}}\right)^{n}=\left(\mathfrak{p} A_{\mathfrak{p}}\right)^{n+1},
$$

because $A_{\mathfrak{p}} \rightarrow B_{\mathfrak{q}}$ is pure, and therefore all ideals in $A_{\mathfrak{p}}$ are contracted. It follows that $f_{1}, \ldots, f_{t}$ generate a $\mathfrak{p} A_{\mathfrak{p}}$-primary ideal in $A_{\mathfrak{p}}$; hence $t>\operatorname{dim} A_{\mathfrak{p}}$. Now, let $\mathrm{F}$ denote the $A_{\mathfrak{p}}$-submodule of $\mathrm{D}_{k}(A)_{\mathfrak{p}}$ generated by $\mathrm{d} f_{1}, \ldots, \mathrm{d} f_{t}$. Because of $\mathrm{U}(\mathfrak{b})=$ $B_{\mathrm{q}} \mathrm{dp} / \mathrm{pD}_{k}(B)_{\mathrm{q}}$ the minimal number of generators of $\mathrm{F}$ is $t$ and the canonical induced map

$$
\mathrm{F} \otimes_{A_{\mathfrak{p}}} \kappa(\mathfrak{p}) \rightarrow \mathrm{D}_{k}(B)_{\mathrm{q}} \otimes_{A_{\mathfrak{b}}} \kappa(\mathfrak{p})
$$

is injective. Therefore it only remains to be shown that the canonical $\boldsymbol{A}_{\mathfrak{p}}$-homomorphism $\psi$ in the composition

$$
\mathrm{F} \rightarrow \mathrm{D}_{k}(A)_{\mathfrak{b}} \stackrel{\psi}{\rightarrow} \mathrm{D}_{k}(B)_{\mathfrak{a}}
$$

factors through $\left(\mathrm{D}_{k}(A)\right)_{\mathrm{p}}^{* *}$. Because of $\operatorname{codim} a B_{\mathrm{q}}=\operatorname{codim}_{Y} Z>2$ there exist elements $g, h \in \mathfrak{a}$ (recall that $\mathrm{D}(\mathfrak{a})$ is the regular locus of $A_{\mathfrak{p}}$ ), which form a prime sequence in $A_{\mathfrak{p}}$ as well as in $B_{\mathfrak{q}}$. Because of

$$
\left(\mathrm{D}_{k}(A)\right)_{\mathfrak{p}}^{* *}=\left(\left(\mathrm{D}_{k}(A)\right)_{\mathfrak{p}}^{* *}\right)_{g} \cap\left(\left(\mathrm{D}_{k}(A)\right)_{\mathfrak{p}}^{* *}\right)_{h}=\left(\mathrm{D}_{k}(A)_{\mathfrak{p}}\right)_{g} \cap\left(\mathrm{D}_{k}(A)_{\mathfrak{p}}\right)_{h}
$$

and

$$
\mathrm{D}_{k}(B)_{\mathrm{q}}=\left(\mathrm{D}_{k}(B)_{\mathrm{q}}\right)_{\mathrm{g}} \cap\left(\mathrm{D}_{k}(B)_{\mathrm{q}}\right)_{h}
$$

the assertion of the proposition follows.

REMARK 1. The condition $\operatorname{codim}_{Y} Z \geqslant 2$ is automatically satisfied, if $\varphi$ is nondegenerate in $q$.

Remark 2. The elements $f_{1}, \ldots, f_{s} \in \mathfrak{p}$ in Proposition 1 may be chosen as part of any given system of generators of $\mathfrak{p}$ or of $\mathfrak{p} A_{\mathfrak{p}}$. In particular, they may be chosen as a system of parameters of $A_{p}$; see the proof of Proposition 1.

REMARK 3. One can replace the hypothesis that $A$ and $B$ are local rings by the assumption that the canonical homomorphism $\mathrm{D}_{k}(A) \rightarrow \mathrm{D}_{k}(B)$ exists. 
REMARK 4. In view of condition (1) at the beginning of this section we recall some criteria for $D_{k}(B)_{a}$ to be reflexive. This is the case, if, for instance, one of the following conditions is satisfied.

(a) $B_{\mathrm{q}}$ is an (abstract) complete intersection which is regular in codimension 2.

(b) The homological codimension of $D_{k}(B)_{a}$ is at least by 2 larger than the dimension of the singular locus of $B_{\mathrm{q}}$.

(c) $B$ is an (analytic) singularity of the cone over a Sègre variety.

If $\operatorname{codim}_{Y} Z \geqslant 2$, the Zariski-Lipman conjecture for $A_{\mathfrak{p}}$ is evidently solvable: If $\left(\mathrm{D}_{k}(A)\right)_{\mathfrak{p}}^{*}$ is free, $A_{\mathfrak{p}}$ is regular.

Proof. If $\left(\mathrm{D}_{k}(A)\right)_{\mathfrak{p}}^{*}$ is free, the differentials $\mathrm{d} f_{1}, \ldots, \mathrm{d} f_{s}$ in Proposition 1 form part of a free basis of $\left(\mathrm{D}_{k}(A)\right)_{\mathfrak{p}}^{* *}$. Hence there exist derivations of $A_{\mathfrak{p}} \delta_{1}, \ldots, \delta_{s}$ with $\delta_{i} f_{j}=\delta_{i j}, i, j=1, \ldots, s$, and $A_{\mathfrak{p}}$ is therefore regular, see [9].

However, we still obtain the solution of the Zariski-Lipman conjecture for $A_{\mathfrak{p}}$, if we require a geometric condition on $Y=\operatorname{Spec} B_{\mathrm{a}}$ (instead of $\operatorname{codim}_{Y} Z>2$ ) in the sense of the following definition; compare also condition $\left(W^{\prime}\right)$ in [2, p. 308]:

Definition. Let Spec $S$ be a noetherian affine scheme. We say that Spec $S$ (or simply $S$ ) is divisorially affine, if Spec $S$ is a (reduced) normal scheme and if the complement of every divisor in Spec $S$, i.e. of every closed pure one-codimensional set is affine.

A noetherian scheme is divisorially affine if and only if all localizations are divisorially affine. It is well known that all (noetherian) factorial domains (even all locally almost factorial rings) and, by (2.7) in [1], all excellent normal domains of dimension 2 are divisorially affine. We now prove

Proposition 2. Let $Y=\operatorname{Spec} B_{\mathfrak{q}}$ be divisorially affine. If $\left(\mathrm{D}_{k}(A)\right)_{\mathfrak{p}}^{*}$ is free, $A_{\mathfrak{p}}$ is regular.

Proof. According to the remarks on purity in $\S 1$ we may assume $V=$ $X \backslash\left\{\mathfrak{p} A_{\mathfrak{p}}\right\}$. Since $A_{\mathfrak{p}} \rightarrow B_{\mathrm{q}}$ is pure, $U=\varphi_{*}^{-1}(V)$ is not affine. $Y=\operatorname{Spec} B_{\mathrm{q}}$ is divisorially affine; it follows that $Z=Y \backslash U$ cannot be of pure codimension 1, i.e. there exists an irreducible component of $Z$ of codimension $>2$. Therefore, let $\mathfrak{q}^{\prime} B_{\mathfrak{q}} \supseteq \mathfrak{p} B_{\mathfrak{q}}$ be a minimal prime ideal of $\mathfrak{p} B_{\mathrm{q}}$ of height $>2$. We consider the local injection $A_{\mathfrak{p}} \rightarrow B_{\mathfrak{q}^{\prime}}$ (which is not necessarily pure!). Because of $\operatorname{codim} \mathfrak{p} B_{\mathfrak{q}^{\prime}}=$ $\operatorname{dim} B_{\mathfrak{q}^{\prime}} \geqslant 2$ the canonical $A_{\mathfrak{p}}$-homomorphism $\mathrm{D}_{k}(A)_{\mathfrak{p}} \rightarrow \mathrm{D}_{k}(B)_{\mathfrak{q}^{\prime}}$ factors through $\left(\mathrm{D}_{k}(A)\right)_{\mathfrak{p}}^{* *}$; see the proof of Proposition 1. Now, the module $\mathrm{U}\left(\mathfrak{p} B_{\mathfrak{q}^{\prime}}\right)=$ $B_{\mathfrak{q}^{\prime}} \mathrm{dp} / \mathfrak{p D} D_{k}(B)_{\mathfrak{q}^{\prime}}$ is not zero, because otherwise $\mathfrak{p} B_{\mathfrak{q}^{\prime}}$ would be nilpotent. Let $f \in \mathfrak{p}$ with $\mathrm{d} f \notin \mathrm{pD}_{k}(B)_{\mathrm{q}^{\prime}}$. It then follows that the differential $\mathrm{d} f$ is part of a free basis of $\left(\mathrm{D}_{k}(A)\right)_{\mathfrak{p}}^{* *}$. Hence there exists a derivation $\delta$ of $A_{\mathfrak{p}}$ with $\delta f=1$. This contradicts the well-known lemma of Zariski. [For, if $\delta f=1$ with $f \in \mathfrak{p}$, then there exists a subring $R \subseteq A_{\hat{p}}$ such that $\hat{A_{\mathfrak{p}}}=R \llbracket f \rrbracket$ is a power series ring in $f$ over $R$; see Theorem 2 in [4]. I Let $\mathrm{c}:=\mathrm{m}_{R} \cdot A_{\mathfrak{p}}$ be the nonmaximal prime ideal in $A_{\hat{\mathfrak{p}}}$. Then $\left(A_{\mathfrak{p}}\right)_{\mathrm{c}}$ is regular by the assumption on the regular locus of the (excellent) domain $A_{\mathfrak{p}}$. Since $R \rightarrow\left(A_{\mathfrak{p}}^{\hat{p}}\right)$ c is faithfully flat, $R$ and $A_{\mathfrak{p}}=R \llbracket f \rrbracket$ are regular.]

We do not know whether the geometric condition on $Y=\operatorname{Spec} B_{\mathrm{q}}$ in Proposition 2 is really necessary. 
3. Applications. Let $k$ be a field of characteristic zero. From Proposition 2 we obtain

COROllaRY 1. Let $A \rightarrow B$ be a pure extension of noetherian $k$-algebras for which the finite differential modules $\mathrm{D}_{k}(A)$ and $\mathrm{D}_{k}(B)$ exist. Let $B$ be divisorially affine and $\mathrm{D}_{k}(B)$ be reflexive. If $\left(\mathrm{D}_{k}(A)\right)_{\mathfrak{p}}^{*}$ is free for a prime ideal $\mathfrak{p} \subseteq A, A_{\mathfrak{p}}$ is regular.

Proof. Let $\mathfrak{m} \supseteq \mathfrak{p}$ be a maximal ideal in $A$. According to the remarks on purity in $\S 1$ there exists a maximal ideal $\mathfrak{M} \subseteq B$ lying over $\mathfrak{m}$ such that the homomorphism $A_{\mathfrak{m}} \rightarrow B_{\mathfrak{M}}$ is pure, too. Therefore we may assume that $A$ and $B$ are local rings. Since there is a prime ideal $\mathfrak{q} \subseteq B$ lying over $\mathfrak{p}$ such that $A_{\mathfrak{p}} \rightarrow B_{\mathfrak{q}}$ is pure, the assertion follows from Proposition 2.

Now, let $G$ be a reductive linear algebraic group acting ( $k$-rationally) on the $k$-algebra $B$. Then the extension $B^{G} \rightarrow B$ is pure, since $B^{G}$ is a direct $B^{G}$-summand of $B$ (via the canonical injection). It is well known that, if $B$ is a finitely generated $k$-algebra, the invariant ring $B^{G}$ is also a finitely generated $k$-algebra. For the theory of linear algebraic groups see [5] and [3], especially $\$ 10$ and the literature given there. From Corollary 1 we obtain immediately

COROLlARY 2. Let $G$ be a reductive linear algebraic group acting ( $k$-rationally) on the noetherian $k$-algebra $B$. Assume that the finite differential modules $\mathrm{D}_{k}(A)$ and $\mathrm{D}_{k}(B)$ exist, where $A:=B^{G}$ denotes the invariant (noetherian) $k$-algebra. Let $B$ be divisorially affine and $\mathrm{D}_{k}(B)$ be reflexive. If $\left(\mathrm{D}_{k}(A)\right)_{\mathfrak{p}}^{*}$ is free for a prime ideal $\mathfrak{p} \subseteq A$, $A_{\mathfrak{p}}$ is regular.

We remark that the hypotheses of Corollary 1 and Corollary 2 on Spec $B$ and $\mathrm{D}_{k}(B)$ are evidently fulfilled, if $B$ is regular.

For finite groups $G$ we obtain the result of (3.1) in [6]:

COROLlary 3. Let $G$ be a finite group of $k$-algebra automorphisms on the noetherian $k$-algebra $B$ and $A:=B^{G}$ be the invariant (noetherian) $k$-algebra. Assume that the finite differential modules $\mathrm{D}_{k}(A)$ and $\mathrm{D}_{k}(B)$ exist. Let $q \subseteq B$ be a prime ideal with the property that $B_{\mathrm{q}}$ is a normal domain and $\mathrm{D}_{k}(B)_{\mathrm{q}}$ is reflexive.

Then there exist elements $f_{1}, \ldots, f_{s} \in \mathfrak{p}:=\mathfrak{q} \cap A, s:=\operatorname{dim} A_{\mathfrak{p}}$, whose differentials $\mathrm{d} f_{1}, \ldots, \mathrm{d} f_{s}$ form part of a minimal system of generators of $\left(\mathrm{D}_{k}(A)\right)_{p}^{* *}$ via the canonical homomorphism $\mathrm{D}_{k}(A)_{\mathfrak{p}} \rightarrow\left(\mathrm{D}_{k}(A)\right)_{\mathfrak{p}}^{* *}$.

Proof. Let $\mathfrak{M} \supseteq q$ be a maximal ideal in $B$ and $\mathfrak{m}:=\mathfrak{M} \cap A$. Because of Proposition 1 it only remains to be shown that the homomorphism $A_{\mathfrak{m}} \rightarrow B_{\mathfrak{R}}$ is nondegenerate in $\mathfrak{q} B_{\mathfrak{M}}$, see also Remark 1 . Since $G$ acts on $B_{\mathfrak{p}}$ with $\left(B_{\mathfrak{p}}\right)^{G}=A_{\mathfrak{p}}$, all maximal ideals in $B_{\mathfrak{p}}$ are of the same height and $\mathfrak{p} B_{\mathfrak{q}}$ is a $q B_{q}$-primary ideal. Therefore the equality

$$
\operatorname{dim} A_{\mathfrak{p}}+\operatorname{dim}\left(B_{\mathfrak{q}} / \mathfrak{p} B_{\mathfrak{q}}\right)=\operatorname{dim} B_{\mathfrak{q}}
$$

holds for trivial reasons.

Remark 5. As it can easily be seen from the proof of Proposition 1, the assumption of Corollary 3 that $k$ is of characteristic zero is quite essential. 
However, the embedding property of Corollary 3 is obtained, for instance, in the analytic case,

(a) if $q \subseteq B$ is assumed to be smooth in the sense of [6, §2], i.e. if $D_{k}(B)_{q}$ is a free module of rank $\operatorname{dim} B_{\mathrm{q}}+\operatorname{dim} B / \mathrm{q}$, and

(b) if $q \subseteq B$ is assumed to be separable, i.e. if $\operatorname{rank}\left(D_{k}(B / q)\right)=\operatorname{dim} B / q$ with card $G$ being a unit in $k$ : In (2.1) of [6] it has been shown that

$$
\mu\left(\mathrm{D}_{k}(B)\right)_{\mathfrak{p}}^{G}>\operatorname{rank}\left(\mathrm{D}_{k}(A)_{\mathfrak{p}}\right)+\mu\left(\mathrm{D}_{A}(B)\right)_{\mathfrak{p}}^{G} .
$$

From the isomorphisms in (2.3) of [6] we obtain

$$
\mu\left(\mathrm{D}_{k}(A)\right)_{\mathfrak{p}}^{* *}>\operatorname{rank}\left(\mathrm{D}_{k}(A)_{\mathfrak{p}}\right)+\mu\left(\operatorname{Coker}\left(\mathrm{D}_{k}(A)\right)_{\mathfrak{p}} \rightarrow\left(\mathrm{D}_{k}(A)\right)_{\mathfrak{p}}^{* *}\right)
$$

where $\mu(-)$ denotes the minimal number of generators. Because of

$$
\begin{aligned}
& \operatorname{rank}\left(\mathrm{D}_{k}(A)_{\mathfrak{p}}\right)=\operatorname{rank}\left(\mathrm{D}_{k}(B)_{\mathfrak{q}}\right)=\operatorname{dim} B_{\mathfrak{q}}+\operatorname{dim} B / \mathfrak{q}=\operatorname{dim} A_{\mathfrak{p}}+\operatorname{dim} A / \mathfrak{p}, \\
& \operatorname{rank}\left(\mathrm{D}_{k}(A / \mathfrak{p})\right)=\operatorname{rank}\left(\mathrm{D}_{k}(B / \mathfrak{q})\right)=\operatorname{dim} B / \mathfrak{q}=\operatorname{dim} A / \mathfrak{p}
\end{aligned}
$$

and the canonical exact sequence of $\kappa(\mathfrak{p})$-vector spaces

$$
\left(\mathfrak{p} / \mathfrak{p}^{2}\right)_{\mathfrak{p}} \rightarrow\left(\mathrm{D}_{k}(A) / \mathfrak{p D} \mathrm{D}_{k}(A)\right)_{\mathfrak{p}} \rightarrow \mathrm{D}_{k}(A / \mathfrak{p})_{(0)} \rightarrow 0
$$

we obtain that there exist $s=\operatorname{dim} A_{\mathfrak{p}}$ elements $f_{1}, \ldots, f_{s} \in \mathfrak{p}$, whose differentials $\mathrm{d} f_{1}, \ldots, \mathrm{d} f_{s}$ form part of a minimal system of generators of $\left(\mathrm{D}_{k}(A)\right)_{\mathfrak{p}}^{* *}$. We remark that in case char $k=0$ the canonical induced map

$$
\mathrm{D}_{k}(A)_{\mathfrak{p}} \otimes_{A_{\mathfrak{p}}} \kappa(\mathfrak{p}) \rightarrow\left(\mathrm{D}_{k}(A)\right)_{\mathfrak{p}}^{* *} \otimes_{A_{\mathfrak{p}}} \kappa(\mathfrak{p})
$$

is even injective, see (2.7) in [6].

The assertion of Proposition 1 has been proved in $\$ 5$ of the author's dissertation Operation von endlichen Gruppen auf Differentialen, Dissertation Universität Osnabrück, Juni 1977 under the assumption that the extension $A_{\mathfrak{p}} \rightarrow B_{\mathfrak{q}}$ is quasifinite. I would like to express my gratitude to my thesis advisor Uwe Storch in Osnabrück for pointing out to me that the result could possibly be generalized to pure extensions.

\section{REFERENCES}

1. J. Bingener and U. Storch, Resträume zu analytischen Mengen in Steinschen Räumen, Math. Ann. 210 (1974), 33-53.

2. A. Grothendieck and J. Dieudonnée, Éléments de géometrie algébrique. IV, Inst. Hautes Études Sci. Publ. Math. 32 (1967).

3. M. Hochster and J. L. Roberts, Rings of invariants of reductive groups acting on regular rings are Cohen-Macaulay, Adv. in Math. 13 (1974), 115-175.

4. J. Lipman, Free derivation modules on algebraic varieties, Amer. J. Math. 87 (1965), 874-898.

5. D. Mumford, Geometric invariant theory, Springer-Verlag, Berlin and New York, 1965.

6. E. Platte, Differentielle Eigenschaften der Invarianten regulärer Algebren, J. Algebra 62 (1980), 1-12.

7. G. Scheja and U. Storch, Über differentielle Abhängigkeit bei Idealen analytischer Algebren, Math. Z. 114 (1970), 101-112.

8. (1972), 137-170. Differentielle Eigenschaften der Lokalisierungen analytischer Algebren, Math. Ann. 197

9. W. V. Vasconcelos, Derivations of commutative noetherian rings, Math. Z. 112 (1969), 229-233.

Fachbereich 3, Universität Osnabrück, Abteilung Vechta, D-2848 Vechta, Federal Republic OF GeRMANY 